\title{
Robust STAP for MIMO Radar Based on Direct Data Domain Approach
}

\author{
Cheng Chen, ${ }^{1}$ Junjie He, ${ }^{2}$ Zeshi Yuan, ${ }^{1}$ Xiaohua $\mathrm{Zhu}^{1}$ and Hongtao $\mathrm{Li}^{1}$ \\ ${ }^{1}$ Nanjing University of Science and Technology, Nanjing, Jiangsu 210094, China \\ ${ }^{2}$ The 28th Research Institute of China Electronics Technology Group Corporation, Nanjing 210094, China
}

Correspondence should be addressed to Xiaohua Zhu; njust_zxh@163.com

Received 3 August 2017; Accepted 18 October 2017; Published 23 November 2017

Academic Editor: Elisa Giusti

Copyright ( 2017 Cheng Chen et al. This is an open access article distributed under the Creative Commons Attribution License, which permits unrestricted use, distribution, and reproduction in any medium, provided the original work is properly cited.

\begin{abstract}
The detection performance of direct data domain $\left(\mathrm{D}^{3}\right)$ space-time adaptive processing (STAP) will be extremely degraded when there are mismatches between the actual and the presumed signal steering vectors. In this paper, a robust $\mathrm{D}^{3}$ STAP method for multiple-input multiple-output (MIMO) radar is developed. The proposed method utilizes the worst-case performance optimization (WCPO) to prevent the target self-nulling effect. An upper bound for the norm of the signal steering vector error is given to ensure that the WCPO problem has an admissible solution. Meanwhile, to obtain better detection performance in the low signal-to-noise ratio (SNR) environment, the proposed method gives a modified objective function to minimize the array noise while mitigating the interferences. Simulation results demonstrate the validity of our proposed method.
\end{abstract}

\section{Introduction}

Space-time adaptive processing (STAP) is a key technology for airborne surveillance radar system to detect moving targets in a strong interference environment [1]. The working principles of STAP can be classified into two categories: statistical STAP and deterministic STAP (also known as direct data domain $\left(\mathrm{D}^{3}\right)$ STAP) [2-7]. In statistical STAP, the covariance matrices of the interference and the clutter environment are usually estimated from the target-free training data, which is collected from range cells adjacent to the range gate under test $[2,3]$. To approach the adequate detection performance, the required amount of the training data is at least twice the STAP filter dimension [8]. However, in the scenarios where the ranges of the interferences change fast or the clutters are nonhomogeneous in the target-free training data, the statistical characteristics of the interference environment are difficult to be precisely estimated. To cope with the aforementioned problems, some methods based on the $\mathrm{D}^{3}$ approach are given in [5-7]. The $\mathrm{D}^{3}$ approach can minimize the interference power while preserving unit gain for the target in a coherent processing interval (CPI). In the
$\mathrm{D}^{3}$ approach, the target signal is firstly removed from the received signal to remain the contributions of the interferences. However, in the practical application, there are mismatches between the actual and the presumed signal steering vectors due to the direction of arrival (DOA) and Doppler frequency errors, imperfect array calibration, and distorted antenna shape [9]. Hence, in such situations, the processed signal also retains the contribution of the target, which may cause the target self-nulling effect.

In this paper, we propose a robust $\mathrm{D}^{3}$ STAP method for colocated multiple-input multiple-output (MIMO) radar, where superior capabilities arouse significant attentions among radar scholars [10-14]. In the presented robust method, the worst-case performance optimization (WCPO) [9] is used as the approach to avoid the target self-nulling effect. An upper bound for the norm of the target steering vector error is given to ensure the existence of a numerical solution for the WCPO problem. For the reason that the statistical characteristics of the array noise are changed in the $\mathrm{D}^{3}$ approach, the $\mathrm{D}^{3}$ approach based on the WCPO cannot minimize the array noise power effectively while mitigating the interferences. Hence, a modified $\mathrm{D}^{3}$ approach is given to 


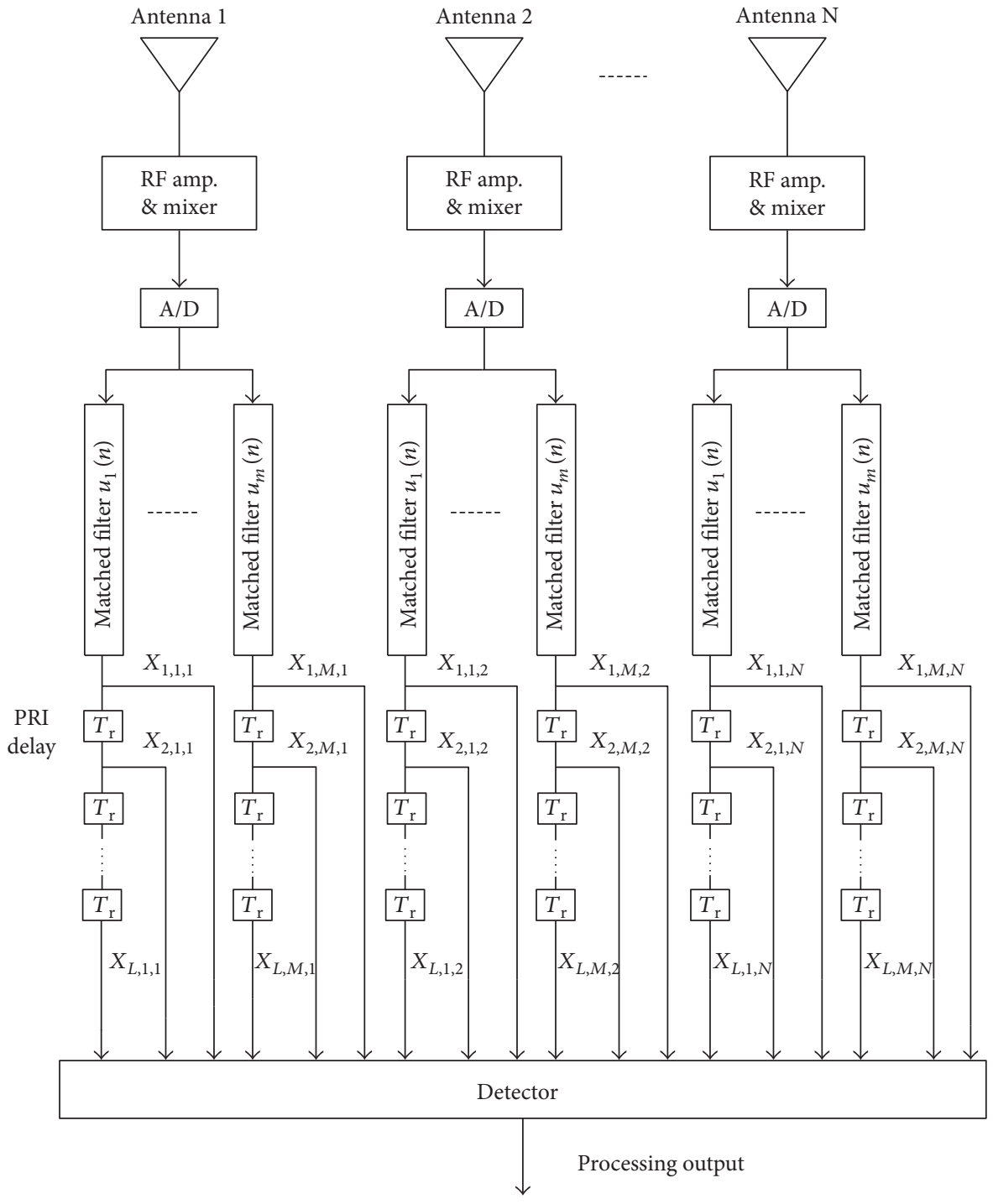

FIgURE 1: Structure of MIMO radar receiver in a CPI.

obtain a better detection performance in the low signal-tonoise ratio (SNR) environment. In the modified method, the objective function of the $\mathrm{D}^{3}$ approach is replaced by a linear combination of the original objective function and the output of the array noise. Simulation results show that the proposed method can avoid the target self-nulling effect and outperforms the conventional robust $\mathrm{D}^{3}$ approach in the low SNR environment.

\section{Signal Model}

Consider a MIMO radar system equipped with a transmit uniform linear array (ULA) of $M$ elements and a receive ULA of $N$ elements. Each transmit array element emits a burst of $L$ pulses in a CPI with a pulse repetition frequency (PRF) $f_{\mathrm{r}}$. The waveforms of $M$ transmit array elements are mutually orthogonal and repeated pulse to pulse. Hence, the waveform of the $i$ th transmit array element in a CPI can be written as [15]

$$
\tilde{u}_{i}(t)=\sum_{l=1}^{L} u_{i}\left(t-l T_{\mathrm{r}}\right), \quad i=1,2, \ldots, M,
$$

where $T_{\mathrm{r}}=1 / f_{\mathrm{r}}$ is the pulse repetition interval (PRI) and $u_{i}(t)$ is the $i$ th orthogonal waveform which satisfies the following property:

$$
\int_{T_{\mathrm{r}}} u_{i}(t) u_{j}^{*}(t) \mathrm{d} t= \begin{cases}0, & i \neq j \\ 1, & i=j\end{cases}
$$

where $(\cdot)^{*}$ denotes the conjugate operator.

Figure 1 depicts the structure of the MIMO radar receiver in a CPI. As is shown, received signals are collected over $M N$ matched filters in $L$ successive pulses. Assume that there are a point target located at angle $\theta_{\mathrm{t}}$ with Doppler frequency $f_{\mathrm{t}}$ and $K$ interferences located at $\theta_{k}(k=1,2, \ldots, K)$ with Doppler frequency $f_{k}(k=1,2, \ldots, K)$ in the range cell under test. Then, the $m$ th matched filter output from the $n$th receive array element at the $l$ th pulse can be represented as [16] 


$$
\begin{aligned}
x_{l, m, n}=\alpha_{\mathrm{t}} \cdot \exp \left\{j 2 \pi \left[\frac{(m-1) d_{\mathrm{t}}}{\lambda} \sin \left(\theta_{\mathrm{t}}\right)+\frac{(n-1) d_{\mathrm{r}}}{\lambda} \sin \left(\theta_{\mathrm{t}}\right)\right.\right. \\
\left.\left.+\frac{(l-1) f_{\mathrm{t}}}{f_{\mathrm{r}}}\right]\right\} \\
+\sum_{k=1}^{K} \alpha_{k} \cdot \exp \left\{j 2 \pi \left[\frac{(m-1) d_{\mathrm{t}}}{\lambda} \sin \left(\theta_{k}\right)\right.\right. \\
\left.\left.+\frac{(n-1) d_{\mathrm{r}}}{\lambda} \sin \left(\theta_{k}\right)+\frac{(l-1) f_{k}}{f_{\mathrm{r}}}\right]\right\}+r_{l, m, n},
\end{aligned}
$$

where $d_{\mathrm{t}}$ is the interspace distance of the adjacent transmit array elements, $d_{\mathrm{r}}$ is the interspace distance of the adjacent receive array elements, $\lambda$ is the carrier wavelength, and $r_{l, m, n}$ is the noise with zero mean and variance $\sigma_{n}^{2}$.

By rearranging $x_{l, m, n}(l=1,2, \ldots, L, m=1,2, \ldots M, n=1$, $2, \ldots, N)$, the match filter outputs can be written in a vectorial form as

$$
\begin{aligned}
\mathbf{x}= & \alpha_{\mathrm{t}} \mathbf{a}_{\mathrm{d}}\left(f_{\mathrm{t}}\right) \otimes \mathbf{a}_{\mathrm{r}}\left(\theta_{\mathrm{t}}\right) \otimes \mathbf{a}_{\mathrm{t}}\left(\theta_{\mathrm{t}}\right) \\
& +\sum_{k=1}^{K} \alpha_{k} \mathbf{a}_{\mathrm{d}}\left(f_{k}\right) \otimes \mathbf{a}_{\mathrm{r}}\left(\theta_{k}\right) \otimes \mathbf{a}_{\mathrm{t}}\left(\theta_{k}\right)+\mathbf{r},
\end{aligned}
$$

where $\mathbf{r}$ is the noise vector, $\otimes$ denotes the Kronecker product, $\mathbf{a}_{\mathrm{d}}(f), \mathbf{a}_{\mathrm{r}}(\theta)$, and $\mathbf{a}_{\mathrm{t}}(\theta)$ are Doppler steering vector, receive spatial steering vector, and transmit steering vector, respectively, which are given by

$$
\begin{aligned}
& \mathbf{a}_{\mathrm{d}}(f)=\left[1, \exp \left(j 2 \pi \frac{f}{f_{\mathrm{r}}}\right), \ldots, \exp \left(j 2 \pi(L-1) \frac{f}{f_{\mathrm{r}}}\right)\right]^{\mathrm{T}} \\
& \mathbf{a}_{\mathrm{r}}(\theta)=\left[1, \exp \left(j 2 \pi \frac{d_{\mathrm{r}}}{\lambda} \sin (\theta)\right), \ldots, \exp \left(j 2 \pi \frac{(N-1) d_{\mathrm{r}}}{\lambda} \sin (\theta)\right)\right]^{\mathrm{T}},
\end{aligned}
$$

$\mathbf{a}_{\mathrm{t}}(\theta)=\left[1, \exp \left(j 2 \pi \frac{d_{\mathrm{t}}}{\lambda} \sin (\theta)\right), \ldots, \exp \left(j 2 \pi \frac{(N-1) d_{\mathrm{t}}}{\lambda} \sin (\theta)\right)\right]^{\mathrm{T}}$

\section{Proposed Method}

In a rapidly changing environment, the $\mathrm{D}^{3}$ STAP outperforms the statistical STAP for MIMO radar, due to the inaccurate interference covariance matrix estimation of the statistical STAP [7]. However, the detection performance of the $\mathrm{D}^{3}$ STAP will be extremely degraded, when there are steering vector errors [6]. Moreover, the statistical characteristics of the array noise are changed in the $\mathrm{D}^{3}$ approach, which will cause an adverse effect on the detection performance in the low signal-to-noise ratio (SNR) environment. To tackle the above problems, we introduce a modified robust $\mathrm{D}^{3}$ STAP for MIMO radar in the following subsections.

3.1. Conventional $D^{3}$ STAP for MIMO Radar. In this subsection, we briefly introduce the $\mathrm{D}^{3}$ STAP for MIMO radar in absence of steering vector errors. The corresponding work can also be found in [7]. Define three complex scalar quantities as follows:

$$
\begin{aligned}
& z_{\mathrm{d}}=\exp \left(j 2 \pi \frac{f_{\mathrm{t}}}{f_{\mathrm{r}}}\right), \\
& z_{\mathrm{r}}=\exp \left(j 2 \pi \frac{d_{\mathrm{r}}}{\lambda} \sin \left(\theta_{\mathrm{t}}\right)\right), \\
& z_{\mathrm{t}}=\exp \left(j 2 \pi \frac{d_{\mathrm{t}}}{\lambda} \sin \left(\theta_{\mathrm{t}}\right)\right),
\end{aligned}
$$

which are determined by the DOA and the Doppler frequency of the target under test. Through removing the contributions of the target, we can obtain the following seven quantities which only contain the interference and the noise contributions:

$$
\begin{aligned}
& x_{l, m, n}-z_{\mathrm{d}}^{-1} x_{l+1, m, n} \quad l=1,2, \ldots, L-1 ; m=1,2, \ldots, M ; \\
& n=1,2, \ldots, N \\
& x_{l, m, n}-z_{\mathrm{t}}^{-1} x_{l, m+1, n} \quad l=1,2, \ldots, L ; m=1,2, \ldots, M-1 ; \\
& n=1,2, \ldots, N \\
& x_{l, m, n}-z_{\mathrm{r}}^{-1} x_{l, m, n+1} \quad l=1,2, \ldots, L ; m=1,2, \ldots, M \text {; } \\
& n=1,2, \ldots, N-1 \text {; } \\
& x_{l, m, n}-z_{\mathrm{d}}^{-1} z_{\mathrm{t}}^{-1} x_{l+1, m+1, n} \quad l=1,2, \ldots, L-1 \text {; } \\
& m=1,2, \ldots, M-1 ; n=1,2, \ldots, N \text {; } \\
& x_{l, m, n}-z_{\mathrm{d}}^{-1} z_{\mathrm{r}}^{-1} x_{l+1, m, n+1} \quad l=1,2, \ldots, L-1 ; m=1,2, \ldots, M \\
& n=1,2, \ldots, N-1 \text {; } \\
& x_{l, m, n}-z_{\mathrm{t}}^{-1} z_{\mathrm{r}}^{-1} x_{l, m+1, n+1} \quad l=1,2, \ldots, L ; m=1,2, \ldots, M-1 \text {; } \\
& n=1,2, \ldots, N-1 \\
& x_{l, m, n}-z_{\mathrm{d}}^{-1} z_{\mathrm{t}}^{-1} z_{\mathrm{r}}^{-1} x_{l+1, m+1, n+1} \quad l=1,2, \ldots, L-1 \text {; } \\
& m=1,2, \ldots, M-1 \text {; } \\
& n=1,2, \ldots, N-1 \text {. }
\end{aligned}
$$

By arranging the relationships in (9) as rows in a linear system matrix $\mathbf{F}$, a weight vector $\mathbf{w}$ of dimension $K_{l} K_{n} K_{m}$ can be designed to minimize the interferences while preserving unit gain for the target [7]. The corresponding problem can be formulated as

$$
\begin{array}{ll}
\min _{\mathbf{w}} & \left\|\mathbf{F}^{*} \mathbf{w}\right\|, \\
\text { s.t. } & \mathbf{w}^{\mathrm{H}} \mathbf{a}^{\left(K_{l} K_{n} K_{m}\right)}\left(f_{\mathrm{t}}, \theta_{\mathrm{t}}\right)=1,
\end{array}
$$

where $(\cdot)^{\mathrm{H}}$ is the conjugate transpose operator and $\|\cdot\|$ denotes the Euclidean norm. $\mathbf{a}^{\left(K_{l} K_{n} K_{m}\right)}\left(f_{\mathrm{t}}, \theta_{\mathrm{t}}\right)=\tilde{\mathbf{a}}_{\mathrm{d}}$ $\left(f_{\mathrm{t}}\right) \otimes \tilde{\mathbf{a}}_{\mathrm{r}}\left(\theta_{\mathrm{t}}\right) \otimes \tilde{\mathbf{a}}_{\mathrm{t}}\left(\theta_{\mathrm{t}}\right)$ is a truncated version of the target steering vector, while $\tilde{\mathbf{a}}_{\mathrm{d}}\left(f_{\mathrm{t}}\right), \quad \tilde{\mathbf{a}}_{\mathrm{r}}\left(\theta_{\mathrm{t}}\right)$, and $\tilde{\mathbf{a}}_{\mathrm{t}}\left(\theta_{\mathrm{t}}\right)$ are the $K_{l} \times 1, K_{n} \times 1$, and $K_{m} \times 1$ truncated versions of the steering vectors $\mathbf{a}_{\mathrm{d}}\left(f_{\mathrm{t}}\right), \mathbf{a}_{\mathrm{r}}\left(\theta_{\mathrm{t}}\right)$, and $\mathbf{a}_{\mathrm{t}}\left(\theta_{\mathrm{t}}\right)$, respectively. Note that $\left\|\mathbf{F}^{*} \mathbf{w}\right\|$ is only minimized (not nulled) when the 
number of the rows in $\mathbf{F}$ is greater or equal to $K_{l} K_{n} K_{m}$, and the degrees of freedom (DOFs) in the temporal and spatial domain are set to $K_{l} \leq(L+1) / 2, K_{n} \leq(N+1) / 2$, and $K_{m} \leq(M+1) / 2$. It is easy to see that problem (10) is equivalent to the following MVDR problem [17]:

$$
\begin{array}{ll}
\min _{\mathbf{w}} & \mathbf{w}^{\mathrm{H}} \mathbf{F}^{\mathrm{T}} \mathbf{F}^{*} \mathbf{w}, \\
\text { s.t. } & \mathbf{w}^{\mathrm{H}} \mathbf{a}^{\left(K_{l} K_{n} K_{m}\right)}\left(f_{\mathrm{t}}, \theta_{\mathrm{t}}\right)=1 .
\end{array}
$$

The solution is given by

$$
\mathbf{w}=\frac{\left(\mathbf{F}^{\mathrm{T}} \mathbf{F}^{*}\right)^{-1} \mathbf{a}^{\left(K_{l} K_{n} K_{m}\right)}\left(f_{\mathrm{t}}, \theta_{\mathrm{t}}\right)}{\left(\mathbf{a}^{\left(K_{l} K_{n} K_{m}\right)}\left(f_{\mathrm{t}}, \theta_{\mathrm{t}}\right)\right)^{\mathrm{H}}\left(\mathbf{F}^{\mathrm{T}} \mathbf{F}^{*}\right)^{-1} \mathbf{a}^{\left(K_{l} K_{n} K_{m}\right)}\left(f_{\mathrm{t}}, \theta_{\mathrm{t}}\right)} .
$$

\section{2. $D^{3}$ STAP for MIMO Radar Based on the WCPO}

3.2.1. Formulation of the Problem Based on the WCPO. When there are mismatches between the actual and the presumed target steering vectors, the linear system matrix $\mathbf{F}$ also contains the contribution of the target. Hence, the solution of problem (10) will cause the target self-nulling effect. In this subsection, a robust $\mathrm{D}^{3}$ STAP method based on the WCPO is presented to prevent the target self-nulling effect. Let $\mathbf{e}$ be a norm-bounded target steering vector error with $\|\mathbf{e}\| \leq \varepsilon$. By employing the WCPO, problem (10) can be reformulated as

$$
\begin{array}{ll}
\min _{\mathbf{w}} & \left\|\mathbf{F}^{*} \mathbf{w}\right\|, \\
\text { s.t. } & \left|\mathbf{w}^{\mathrm{H}}\left(\mathbf{a}^{\left(K_{l} K_{n} K_{m}\right)}\left(f_{\mathrm{t}}, \theta_{\mathrm{t}}\right)+\mathbf{e}\right)\right| \geq 1 .
\end{array}
$$

According to the derivation in [9], problem (13) is equivalent to the following problem:

$$
\begin{array}{ll}
\min _{\mathbf{w}} & \left\|\mathbf{F}^{*} \mathbf{w}\right\|, \\
\text { s.t. } & \mathbf{w}^{\mathrm{H}} \mathbf{a}^{\left(K_{l} K_{n} K_{m}\right)}\left(f_{\mathrm{t}}, \theta_{\mathrm{t}}\right)-\varepsilon\|\mathbf{w}\| \geq 1, \\
& \operatorname{Im}\left\{\mathbf{w}^{\mathrm{H}} \mathbf{a}^{\left(K_{l} K_{n} K_{m}\right)}\left(f_{\mathrm{t}}, \theta_{\mathrm{t}}\right)\right\}=0,
\end{array}
$$

where $\operatorname{Im}\{\cdot\}$ denotes the imaginary part of the matrix in the bracket. The problem stated in (14) is convex, which can be easily solved by the convex optimization toolbox CVX [18]. In the next subsection, we will discuss the upper bound for the norm of the signal steering vector error.

3.2.2. Upper Bound for the Norm of the Signal Steering Vector Error. Let $\mathbf{e}_{\mathrm{t}}, \mathbf{e}_{\mathrm{r}}$, and $\mathbf{e}_{\mathrm{d}}$ be the error vectors of the transmit spatial steering vector, receive spatial steering vector, and the Doppler steering vector, respectively. Then, the actual signal steering vector can be written as

$$
\begin{aligned}
& \left(\tilde{\mathbf{a}}_{\mathrm{d}}\left(f_{\mathrm{t}}\right)+\mathbf{e}_{\mathrm{d}}\right) \otimes\left(\tilde{\mathbf{a}}_{\mathrm{r}}\left(\theta_{\mathrm{t}}\right)+\mathbf{e}_{\mathrm{r}}\right) \otimes\left(\tilde{\mathbf{a}}_{\mathrm{t}}\left(\theta_{\mathrm{t}}\right)+\mathbf{e}_{\mathrm{t}}\right) \\
= & \left(\tilde{\mathbf{a}}_{\mathrm{d}}\left(f_{\mathrm{t}}\right) \otimes \tilde{\mathbf{a}}_{\mathrm{r}}\left(\theta_{\mathrm{t}}\right)+\tilde{\mathbf{a}}_{\mathrm{d}}\left(f_{\mathrm{t}}\right) \otimes \mathbf{e}_{\mathrm{r}}+\mathbf{e}_{\mathrm{d}} \otimes \tilde{\mathbf{a}}_{\mathrm{r}}\left(\theta_{\mathrm{t}}\right)\right. \\
& \left.+\mathbf{e}_{\mathrm{d}} \otimes \mathbf{e}_{\mathrm{r}}\right) \otimes\left(\tilde{\mathbf{a}}_{\mathrm{t}}\left(\theta_{\mathrm{t}}\right)+\mathbf{e}_{\mathrm{t}}\right) \\
= & \tilde{\mathbf{a}}_{\mathrm{d}}\left(f_{\mathrm{t}}\right) \otimes \tilde{\mathbf{a}}_{\mathrm{r}}\left(\theta_{\mathrm{t}}\right) \otimes \tilde{\mathbf{a}}_{\mathrm{t}}\left(\theta_{\mathrm{t}}\right)+\tilde{\mathbf{a}}_{\mathrm{d}}\left(f_{\mathrm{t}}\right) \otimes \tilde{\mathbf{a}}_{\mathrm{r}}\left(\theta_{\mathrm{t}}\right) \otimes \mathbf{e}_{\mathrm{t}} \\
& +\tilde{\mathbf{a}}_{\mathrm{d}}\left(f_{\mathrm{t}}\right) \otimes \mathbf{e}_{\mathrm{r}} \otimes \tilde{\mathbf{a}}_{\mathrm{t}}\left(\theta_{\mathrm{t}}\right)+\tilde{\mathbf{a}}_{\mathrm{d}}\left(f_{\mathrm{t}}\right) \otimes \mathbf{e}_{\mathrm{r}} \otimes \mathbf{e}_{\mathrm{t}} \\
& +\mathbf{e}_{\mathrm{d}} \otimes \tilde{\mathbf{a}}_{\mathrm{r}}\left(\theta_{\mathrm{t}}\right) \otimes \tilde{\mathbf{a}}_{\mathrm{t}}\left(\theta_{\mathrm{t}}\right)+\mathbf{e}_{\mathrm{d}} \otimes \tilde{\mathbf{a}}_{\mathrm{r}}\left(\theta_{\mathrm{t}}\right) \otimes \mathbf{e}_{\mathrm{t}}+\mathbf{e}_{\mathrm{d}} \otimes \mathbf{e}_{\mathrm{r}} \otimes \tilde{\mathbf{a}}_{\mathrm{t}}\left(\theta_{\mathrm{t}}\right) \\
& +\mathbf{e}_{\mathrm{d}} \otimes \mathbf{e}_{\mathrm{r}} \otimes \mathbf{e}_{\mathrm{t}} .
\end{aligned}
$$

Inspecting on (15), the signal steering vector error is

$$
\begin{aligned}
\mathbf{e}= & \tilde{\mathbf{a}}_{\mathrm{d}}\left(f_{\mathrm{t}}\right) \otimes \tilde{\mathbf{a}}_{\mathrm{r}}\left(\theta_{\mathrm{t}}\right) \otimes \mathbf{e}_{\mathrm{t}}+\tilde{\mathbf{a}}_{\mathrm{d}}\left(f_{\mathrm{t}}\right) \otimes \mathbf{e}_{\mathrm{r}} \otimes \tilde{\mathbf{a}}_{\mathrm{t}}\left(\theta_{\mathrm{t}}\right)+\tilde{\mathbf{a}}_{\mathrm{d}}\left(f_{\mathrm{t}}\right) \otimes \mathbf{e}_{\mathrm{r}} \otimes \mathbf{e}_{\mathrm{t}} \\
& +\mathbf{e}_{\mathrm{d}} \otimes \tilde{\mathbf{a}}_{\mathrm{r}}\left(\theta_{\mathrm{t}}\right) \otimes \tilde{\mathbf{a}}_{\mathrm{t}}\left(\theta_{\mathrm{t}}\right)+\mathbf{e}_{\mathrm{d}} \otimes \tilde{\mathbf{a}}_{\mathrm{r}}\left(\theta_{\mathrm{t}}\right) \otimes \mathbf{e}_{\mathrm{t}}+\mathbf{e}_{\mathrm{d}} \otimes \mathbf{e}_{\mathrm{r}} \otimes \tilde{\mathbf{a}}_{\mathrm{t}}\left(\theta_{\mathrm{t}}\right) \\
& +\mathbf{e}_{\mathrm{d}} \otimes \mathbf{e}_{\mathrm{r}} \otimes \mathbf{e}_{\mathrm{t}} .
\end{aligned}
$$

Assume that the transmit spatial steering vector error $\mathbf{e}_{t}$, the receive spatial steering vector error $\mathbf{e}_{\mathrm{r}}$, and the Doppler steering vector error $\mathbf{e}_{\mathrm{d}}$ are norm-bounded with $\left\|\mathbf{e}_{\mathrm{t}}\right\| \leq \varepsilon_{\mathrm{t}}$, $\|$ $\mathbf{e}_{\mathrm{r}} \| \leq \varepsilon_{\mathrm{r}}$, and $\left\|\mathbf{e}_{\mathrm{d}}\right\| \leq \varepsilon_{\mathrm{d}}$. Then, the upper bound for the norm of $\mathbf{e}$ is given by

$$
\begin{aligned}
\|\mathbf{e}\| \leq & \left\|\tilde{\mathbf{a}}_{\mathrm{d}}\left(f_{\mathrm{t}}\right) \otimes \tilde{\mathbf{a}}_{\mathrm{r}}\left(\theta_{\mathrm{t}}\right) \otimes \mathbf{e}_{\mathrm{t}}\right\|+\left\|\tilde{\mathbf{a}}_{\mathrm{d}}\left(f_{\mathrm{t}}\right) \otimes \mathbf{e}_{\mathrm{r}} \otimes \tilde{\mathbf{a}}_{\mathrm{t}}\left(\theta_{\mathrm{t}}\right)\right\| \\
& +\left\|\tilde{\mathbf{a}}_{\mathrm{d}}\left(f_{\mathrm{t}}\right) \otimes \mathbf{e}_{\mathrm{r}} \otimes \mathbf{e}_{\mathrm{t}}\right\|+\left\|\mathbf{e}_{\mathrm{d}} \otimes \tilde{\mathbf{a}}_{\mathrm{r}}\left(\theta_{\mathrm{t}}\right) \otimes \tilde{\mathbf{a}}_{\mathrm{t}}\left(\theta_{\mathrm{t}}\right)\right\| \\
& +\left\|\mathbf{e}_{\mathrm{d}} \otimes \tilde{\mathbf{a}}_{\mathrm{r}}\left(\theta_{\mathrm{t}}\right) \otimes \mathbf{e}_{\mathrm{t}}\right\|+\left\|\mathbf{e}_{\mathrm{d}} \otimes \mathbf{e}_{\mathrm{r}} \otimes \tilde{\mathbf{a}}_{\mathrm{t}}\left(\theta_{\mathrm{t}}\right)\right\| \\
& +\left\|\mathbf{e}_{\mathrm{d}} \otimes \mathbf{e}_{\mathrm{r}} \otimes \mathbf{e}_{\mathrm{t}}\right\| \\
\leq & \sqrt{K_{l} K_{n}} \varepsilon_{\mathrm{t}}+\sqrt{K_{l} K_{m}} \varepsilon_{\mathrm{r}}+\sqrt{K_{l}} \varepsilon_{\mathrm{t}} \varepsilon_{\mathrm{r}}+\sqrt{K_{n} K_{m}} \varepsilon_{\mathrm{d}} \\
& +\sqrt{K_{n}} \varepsilon_{\mathrm{d}} \varepsilon_{\mathrm{t}}+\sqrt{K_{m}} \varepsilon_{\mathrm{d}} \varepsilon_{\mathrm{r}}+\varepsilon_{\mathrm{d}} \varepsilon_{\mathrm{r}} \varepsilon_{\mathrm{t}} \\
= & \varepsilon_{\mathrm{e}} .
\end{aligned}
$$

Additionally, to ensure that problem (14) has an admissible solution, the norm of the signal steering vector error also has an upper bound as follows [6]:

$$
\|\mathbf{e}\| \leq \frac{K_{l} K_{n} K_{m}-1}{\sqrt{K_{l} K_{n} K_{m}}} .
$$

Hence, the upper bound for the norm of the signal steering vector error $\boldsymbol{e}$ can be found as

$$
\varepsilon_{\max }=\min \left(\frac{K_{l} K_{n} K_{m}-1}{\sqrt{K_{l} K_{n} K_{m}}}, \varepsilon_{\mathrm{e}}\right)
$$

3.3. Modified Robust $D^{3}$ STAP for MIMO Radar. The output signal-to-interference-plus-noise ratio (SINR) of the $\mathrm{D}^{3}$ STAP for MIMO radar is defined by

$$
\operatorname{SINR}=10 \log _{10} \frac{\alpha_{\mathrm{t}}^{2}\left|\mathbf{w}^{\mathrm{H}} \mathbf{a}^{\left(K_{l} K_{n} K_{m}\right)}\left(f_{\mathrm{t}}, \theta_{\mathrm{t}}\right)\right|^{2}}{\mathbf{w}^{\mathrm{H}} \mathbf{R}_{\mathrm{i}+n} \mathbf{w}},
$$


where $\mathbf{R}_{\mathrm{i}+n}$ is the interference-plus-noise covariance matrix, which is given by

$$
\begin{aligned}
\mathbf{R}_{\mathrm{i}+n}= & \sum_{k=1}^{K} \alpha_{k}^{2} \mathbf{a}^{\left(K_{l} K_{n} K_{m}\right)}\left(f_{k}, \theta_{k}\right)\left(\mathbf{a}^{\left(K_{l} K_{n} K_{m}\right)}\left(f_{k}, \theta_{k}\right)\right)^{\mathrm{H}} \\
& +\sigma_{\mathrm{n}}^{2} \mathbf{I}_{K_{l} K_{n} K_{m}} .
\end{aligned}
$$

We can clearly see from (20) and (21) that the average noise power of the system output is $\sigma_{\mathrm{n}}^{2}\|\mathbf{w}\|^{2}$. However, the weight vector $\mathbf{w}$ is designed to minimize the interference and noise contributions in the linear system matrix F. And the statistical characteristics of the noise contributions in the linear system matrix $\mathbf{F}$ are determined by the relationships in (9). For example, one of the noise vectors in the linear system matrix $\mathbf{F}$ can be represented as

$$
\begin{aligned}
\mathbf{n}_{1}= & {\left[r_{1,1,1}-z_{\mathrm{d}}^{-1} r_{2,1,1}, \ldots, r_{1, K_{m}, 1}-z_{\mathrm{d}}^{-1} r_{2, K_{m}, 1},\right.} \\
& r_{1,1,2}-z_{\mathrm{d}}^{-1} r_{2,1,2}, \ldots, r_{1, K_{m}, K_{n}}-z_{\mathrm{d}}^{-1} r_{2, K_{m}, K_{n}}, \\
& r_{2,1,1}-z_{\mathrm{d}}^{-1} r_{3,1,1}, \ldots, r_{2, K_{m}, 1}-z_{\mathrm{d}}^{-1} r_{3, K_{m}, 1}, \\
& r_{2,1,2}-z_{\mathrm{d}}^{-1} r_{3,1,2}, \ldots, r_{2, K_{m}, K_{n}}-z_{\mathrm{d}}^{-1} r_{3, K_{m}, K_{n}}, \ldots, \\
& r_{K_{l}, 1,1}-z_{\mathrm{d}}^{-1} r_{K_{l}+1,1,1}, \ldots, r_{K_{l}, K_{m}, 1}-z_{\mathrm{d}}^{-1} r_{K_{l}+1, K_{m}, 1}, \\
& \left.r_{K_{l}, 1,2}-z_{\mathrm{d}}^{-1} r_{K_{l}+1,1,2}, \ldots, r_{K_{l}, K_{m}, K_{n}}-z_{\mathrm{d}}^{-1} r_{K_{l}+1, K_{m}, K_{n}}\right] .
\end{aligned}
$$

From (22), it is easy to see that the noise component $r_{1,1,1}-z_{\mathrm{d}}^{-1} r_{2,1,1}$ is correlated with the other noise component $r_{2,1,1}-z_{\mathrm{d}}^{-1} r_{3,1,1}$. Therefore, the noise vector of any row of the linear system matrix $\mathbf{F}$ does not obey the distribution of zero mean and covariance matrix $\sigma_{n}^{2} \mathbf{I}_{K_{l} K_{n} K_{m}}$. In this situation, minimizing the objective function of problem (14) cannot ensure the minimum array noise power. To minimize array noise power while mitigating the interferences, we reformulate the robust $\mathrm{D}^{3}$ STAP problem as follows:

$$
\begin{array}{ll}
\min _{\mathbf{w}} & \eta\left\|\mathbf{F}^{*} \mathbf{w}\right\|+(1-\eta)\|\mathbf{w}\| \\
\text { s.t. } & \mathbf{w}^{\mathrm{H}} \mathbf{a}^{\left(K_{l} K_{n} K_{m}\right)}\left(f_{\mathrm{t}}, \theta_{\mathrm{t}}\right)-\varepsilon\|\mathbf{w}\| \geq 1, \\
& \operatorname{Im}\left\{\mathbf{w}^{\mathrm{H}} \mathbf{a}^{\left(K_{l} K_{n} K_{m}\right)}\left(f_{\mathrm{t}}, \theta_{\mathrm{t}}\right)\right\}=0 .
\end{array}
$$

where $\eta \in[0,1]$ is a parameter which balances the weight between the performance of mitigating interferences and that of mitigating noises.

Inspecting on (23), the first and the second parts of the objective function are used to minimize the power of the interferences and array noise, respectively. When $\eta$ is equal to one, problem (23) is equivalent to problem (14). And when $\eta$ is equal to zero, the output signal power spectrum can only preserve a gain for the target without mitigating the interferences. Hence, it is necessary to select an appropriate $\eta$ to solve problem (23). Generally, the value of $\eta$ is proportional to the interference-to-interference-plus-noise ratio. The solution of problem (23) can also be solved by the convex optimization toolbox CVX.

\section{Simulations}

In this section, serval examples are given to investigate the performances of the proposed methods. Consider that the transmit and the receive ULAs are equipped with $M=5$ elements and $N=5$ elements. The interspace distance of the adjacent receive array elements is half the carrier wavelength $\lambda / 2$, and the interspace distance of the adjacent transmit array elements is given as $d_{\mathrm{t}}=5 d_{\mathrm{r}}$. The PRF is $f_{\mathrm{r}}=1150 \mathrm{~Hz}$, and the number of pulses in a CPI is $L=65$. The DOFs of the proposed methods are set to $K_{l}=33, K_{m}=3$, and $K_{n}=3$. The upper bound for the norm of the signal steering vector error in (14) and (22) is $\varepsilon=0.5 \sqrt{K_{l} K_{m} K_{n}}$. In the range cell under test, we suppose that the presumed target is located at $\theta_{\mathrm{t}}=30^{\circ}$ with Doppler frequency $f_{\mathrm{t}}=100 \mathrm{~Hz}$. There are also two interferences located at $\theta_{1}=-10^{\circ}$ with Doppler frequency $f_{\mathrm{t}}=-150 \mathrm{~Hz}$ and $\theta_{2}=65^{\circ}$ with Doppler frequency $f_{2}=200 \mathrm{~Hz}$. The interference-to-noise ratio (INR) is assumed to be $20 \mathrm{~dB}$ for both interferences. And in the following examples, $\eta=0.001$ is chosen for the modified robust $\mathrm{D}^{3}$ STAP.

Example 1. In the first example, the SNR is assumed to be $10 \mathrm{~dB}$ and there are no mismatches between the presumed and the actual signal steering vectors. Figures 2(a) and 2(b) depict the two-dimensional adaptive weight patterns for the conventional $\mathrm{D}^{3}$ STAP and the modified robust $\mathrm{D}^{3}$ STAP, respectively. As shown in Figure 2, both the adaptive weight patterns can form a gain in the target position and place nulls in the interference positions. In addition, we can see that the sidelobe of the adaptive weight pattern obtained by the modified robust $\mathrm{D}^{3}$ STAP is lower than that of the other.

Example 2. In this example, the signal steering vector error is considered. The SNR is assumed to be $10 \mathrm{~dB}$, and the actual target is located at $\tilde{\theta}_{\mathrm{t}}=29.5^{\circ}$ with Doppler frequency $\tilde{f}_{\mathrm{t}}=95 \mathrm{~Hz}$. From Figures $3(\mathrm{a})$ and $3(\mathrm{~b})$, both the adaptive weight patterns place nulls in the interference positions. However, the adaptive weight pattern obtained by the conventional $\mathrm{D}^{3}$ STAP places a null in the actual target position while the adaptive weight pattern obtained by the modified robust $\mathrm{D}^{3}$ STAP preserves a gain in the actual target position.

Example 3. In this example, the output SINRs versus the input SNR are shown in Figures 4(a) and 4(b). The actual target position for Figure 4(b) is the same as that in Example 2. For a comparison, the loaded $\mathrm{D}^{3}$ approach is also given in the simulations, which is a robust method to design the weight vector of (11) like the loaded SMI (LSMI) [19]. The fixed diagonal loading parameter $\gamma=10$ is chosen for the loaded $\mathrm{D}^{3}$ STAP. From Figures 4(a) and 4(b), we can see that the modified robust $\mathrm{D}^{3}$ STAP shows better performance than other methods. This improvement is especially remarkable when the SNR is less than $0 \mathrm{~dB}$.

\section{Conclusion}

In this paper, a robust $\mathrm{D}^{3}$ STAP method for MIMO radar has been presented. The proposed method uses the WCPO 


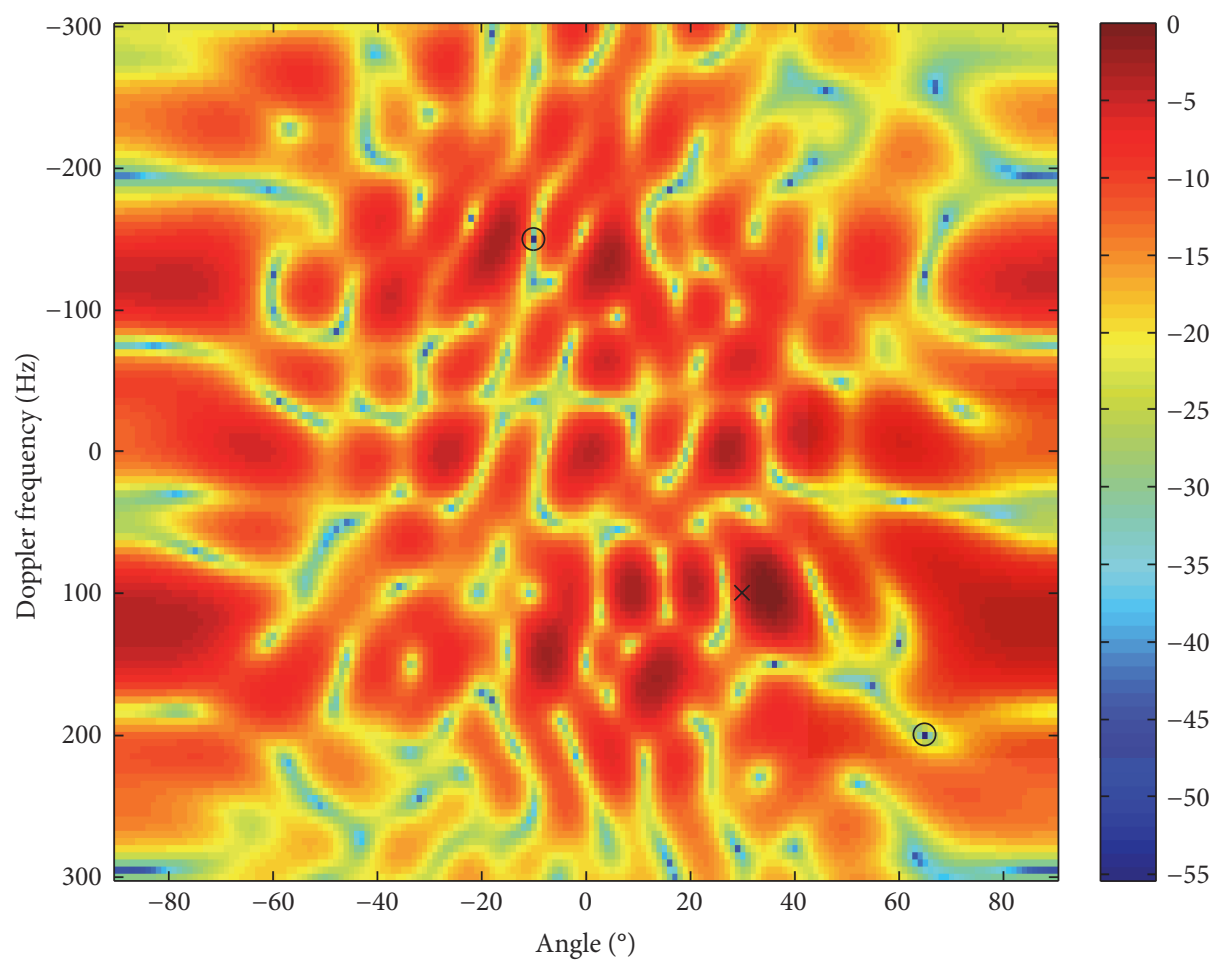

$\times$ True target location

Interference location

(a) The conventional $\mathrm{D}^{3}$ STAP

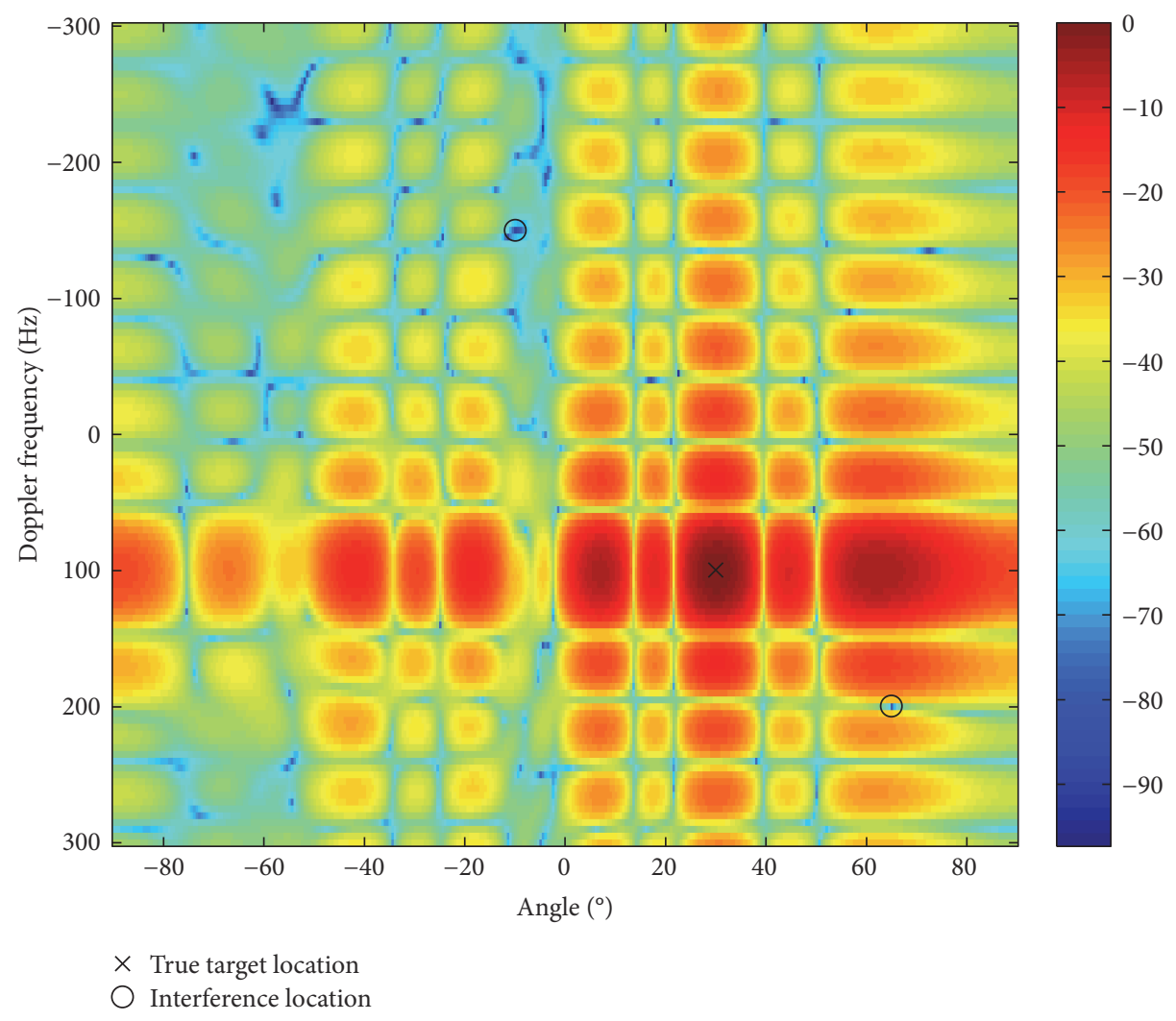

(b) The modified robust $\mathrm{D}^{3}$ STAP

FIgURe 2: Adaptive weight patterns without signal steering vector error. 


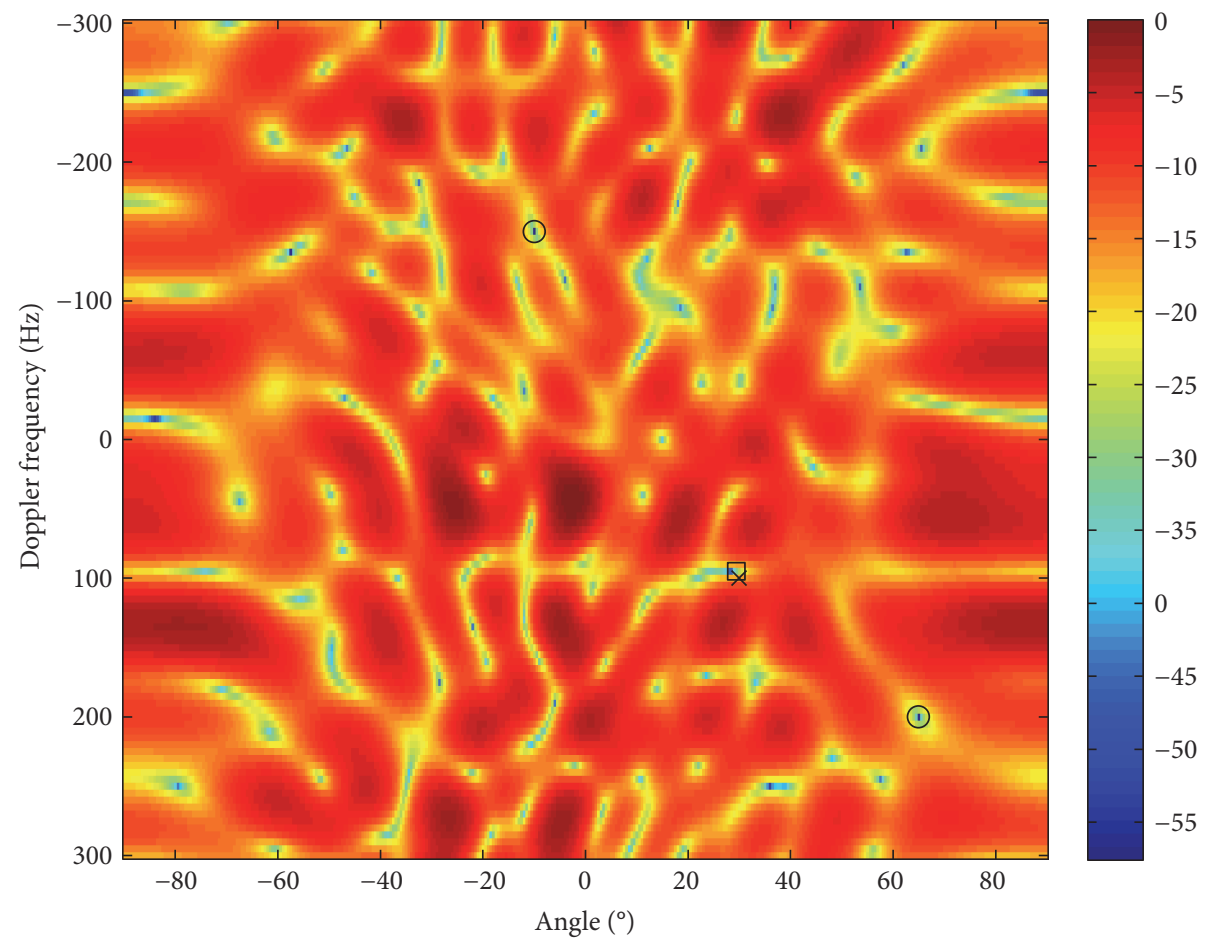
$\times$ Presumed target location
$\square$ True target location
$\bigcirc$ Interference location

(a) The conventional $\mathrm{D}^{3}$ STAP

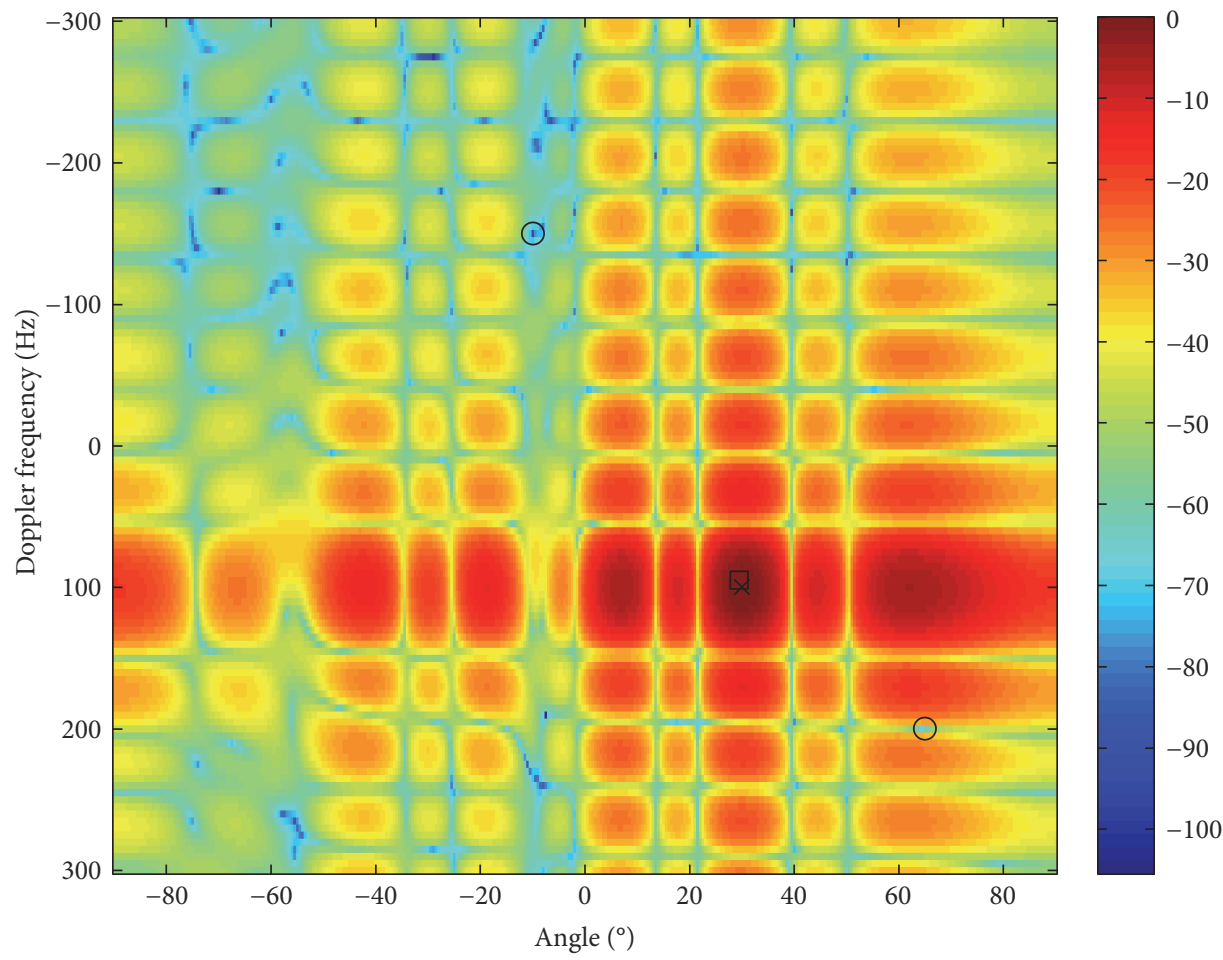
$\times$ Presumed target location
$\square$ True target location
$\bigcirc$ Interference location

(b) The modified robust $\mathrm{D}^{3}$ STAP

FIGURE 3: Adaptive weight patterns with signal steering vector error. 


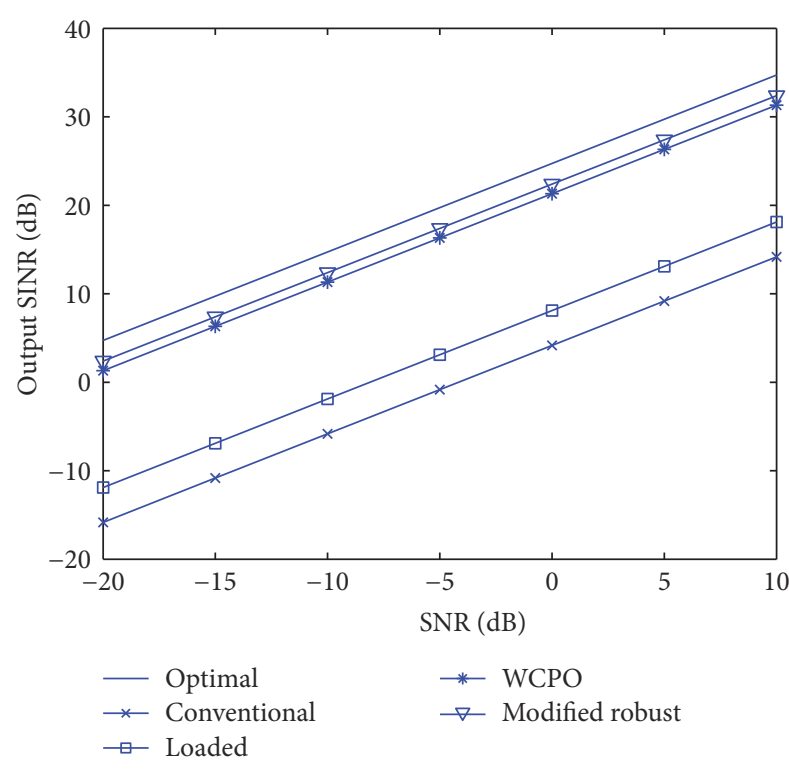

(a) Without signal steering vector error

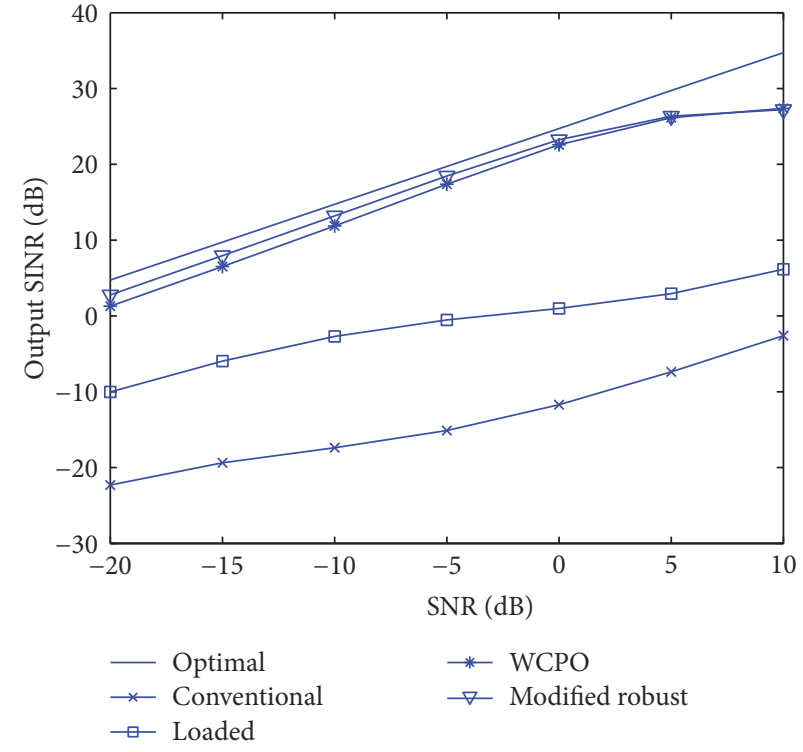

(b) With signal steering vector error

FIgURE 4: Output SINR versus input SNR.

to avoid the target self-nulling effect. Moreover, to improve the output SINR, a modified objective function is proposed to minimize the array noise output while mitigating the interferences. Simulation results show that the proposed method can prevent the target self-nulling effect and provide higher output SINR than other methods in the low SNR environment.

\section{Conflicts of Interest}

The authors declare that there is no conflict of interests regarding the publication of this paper.

\section{Acknowledgments}

This work was supported by the National Natural Science Foundation of China (Grant no. 61401204), Postdoctoral Science Foundation of Jiangsu Province (Grant no. 1501104C), and Technology Research and Development Program of Jiangsu Province (Grant no. BY2015004-03).

\section{References}

[1] H. Wang and L. Cai, "On adaptive spatial-temporal processing for airborne surveillance radar systems," IEEE Transactions on Aerospace and Electronic Systems, vol. 30, no. 3, pp. 660-670, 1994.

[2] H. Jie, F. Zheng, and M. Lun, "Reduced-dimension clutter suppression method for airborne multiple-input multipleoutput radar based on three iterations," IET Radar, Sonar \& Navigation, vol. 9, no. 3, pp. 249-254, 2015.

[3] Y. Wu, T. Wang, J. Wu, and J. Duan, "Training sample selection for space-time adaptive processing in heterogeneous environments," IEEE Geoscience and Remote Sensing Letters, vol. 12, no. 4, pp. 691-695, 2015.

[4] Z. Yang, R. Fa, Y. Qin, X. Li, and H. Wang, "Direct data domain sparsity-based STAP utilizing subaperture smoothing techniques," International Journal of Antennas and Propagation, vol. 2015, Article ID 171808, 10 pages, 2015.

[5] T. K. Sarkar, H. Wang, S. Park et al., "A deterministic leastsquares approach to space-time adaptive processing (STAP)," IEEE Transactions on Antennas and Propagation, vol. 49, no. 1, pp. 91-103, 2001.

[6] D. Cristallini and W. Burger, "A robust direct data domain approach for STAP," IEEE Transactions on Signal Processing, vol. 60 , no. 3, pp. 1283-1294, 2012.

[7] M. Ahmadi, K. Mohamedpour, and F. Keshvari, "Direct data domain $\left(\mathrm{D}^{3}\right)$ approach for space-time adaptive processing in colocated MIMO radar," in 2013 21st Iranian Conference on Electrical Engineering (ICEE), pp. 1-4, Mashhad, Iran, September 2013.

[8] I. S. Reed, J. D. Mallet, and L. E. Brennan, "Rapid convergence rate in adaptive arrays," IEEE Transactions on Aerospace and Electronic Systems, vol. AES-10, no. 6, pp. 853-863, 1974.

[9] S. A. Vorobyov, A. B. Gershman, and Z. Luo, "Robust adaptive beamforming using worst-case performance optimization: a solution to the signal mismatch problem," IEEE Transactions on Signal Processing, vol. 51, no. 2, pp. 313-324, 2003.

[10] J. Li and P. Stoica, "MIMO radar with colocated antennas," IEEE Signal Processing Magazine, vol. 24, no. 5, pp. 106-114, 2007.

[11] X. W. N. Tong, Y. Zhang, G. Hu, and X. He, "Multiple-input multiple-output radar super-resolution three-dimensional imaging based on a dimension-reduction compressive sensing," IET Radar, Sonar \& Navigation, vol. 10, no. 4, pp. 757-764, 2016.

[12] N. Pandey and L. P. Roy, "Convex optimization based transmit beampattern synthesis for MIMO radar," Electronics Letters, vol. 52, no. 9, pp. 761-763, 2016.

[13] B. Jiu, H. Liu, X. Wang, L. Zhang, Y. Wang, and B. Chen, "Knowledge-based spatial-temporal hierarchical MIMO radar waveform design method for target detection in heterogeneous clutter zone," IEEE Transactions on Signal Processing, vol. 63, no. 3, pp. 543-554, 2015. 
[14] Y. Li, S. Gu, and N. Zheng, "MIMO radar transmit beampattern design for DOA estimation with sidelobe suppression," International Journal of Antennas and Propagation, vol. 2016, Article ID 1512843, 10 pages, 2016.

[15] B. Tang and J. Tang, "Joint design of transmit waveforms and receive filters for MIMO radar space-time adaptive processing," IEEE Transactions on Signal Processing, vol. 64, no. 18, pp. 4707-4722, 2016.

[16] W. Wang, Z. Chen, X. Li, and B. Wang, "Space time adaptive processing algorithm for multiple-input-multiple-output radar based on Nyström method," IET Radar, Sonar \& Navigation, vol. 10, no. 3, pp. 459-467, 2016.

[17] J. Capon, "High-resolution frequency-wavenumber spectrum analysis," Proceedings of the IEEE, vol. 57, no. 8, pp. 14081418, 1969.

[18] S. Boyd and L. Vandenberghe, Convex Optimization, Cambridge University Press, 2004.

[19] O. Besson and F. Vincent, "Performance analysis of beamformers using generalized loading of the covariance matrix in the presence of random steering vector errors," IEEE Transactions on Signal Processing, vol. 53, no. 2, pp. 452-459, 2005. 


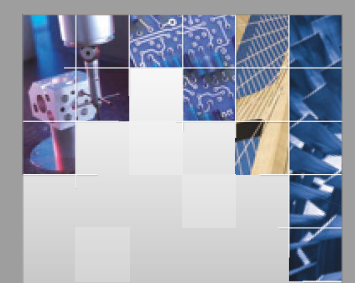

\section{Enfincering}
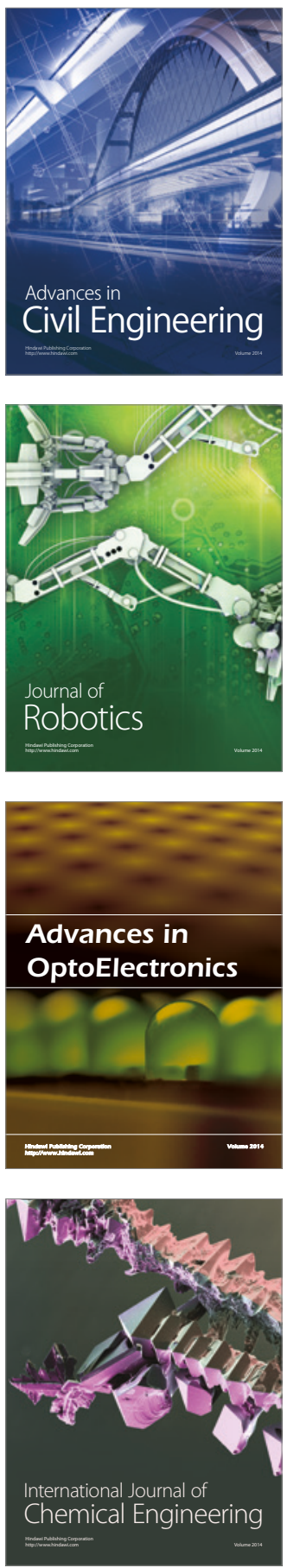

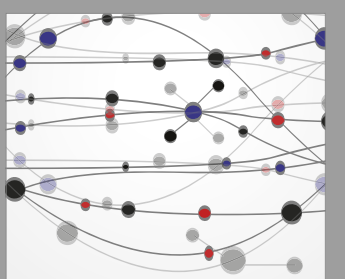

The Scientific World Journal

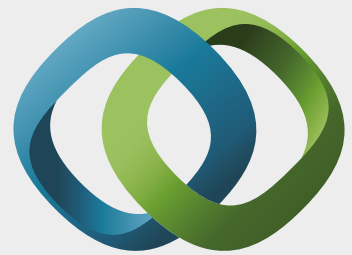

\section{Hindawi}

Submit your manuscripts at

https://www.hindawi.com
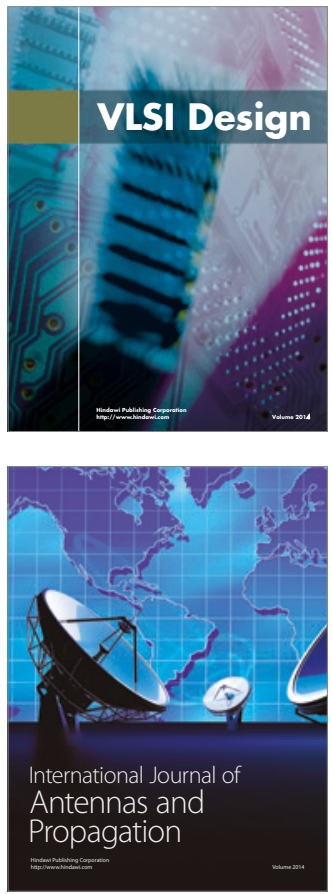

\section{Rotating}

Machinery
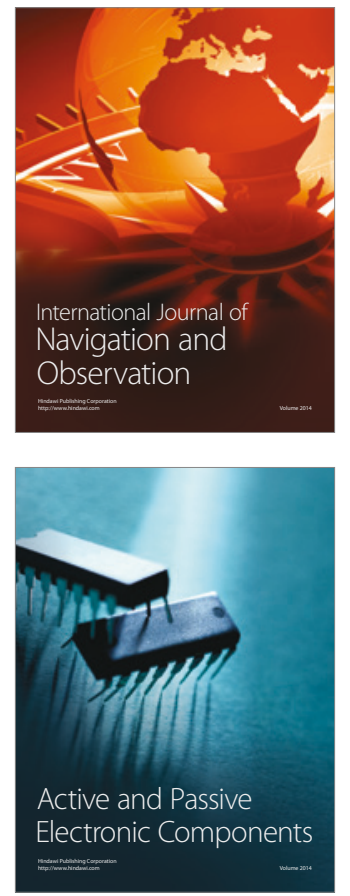
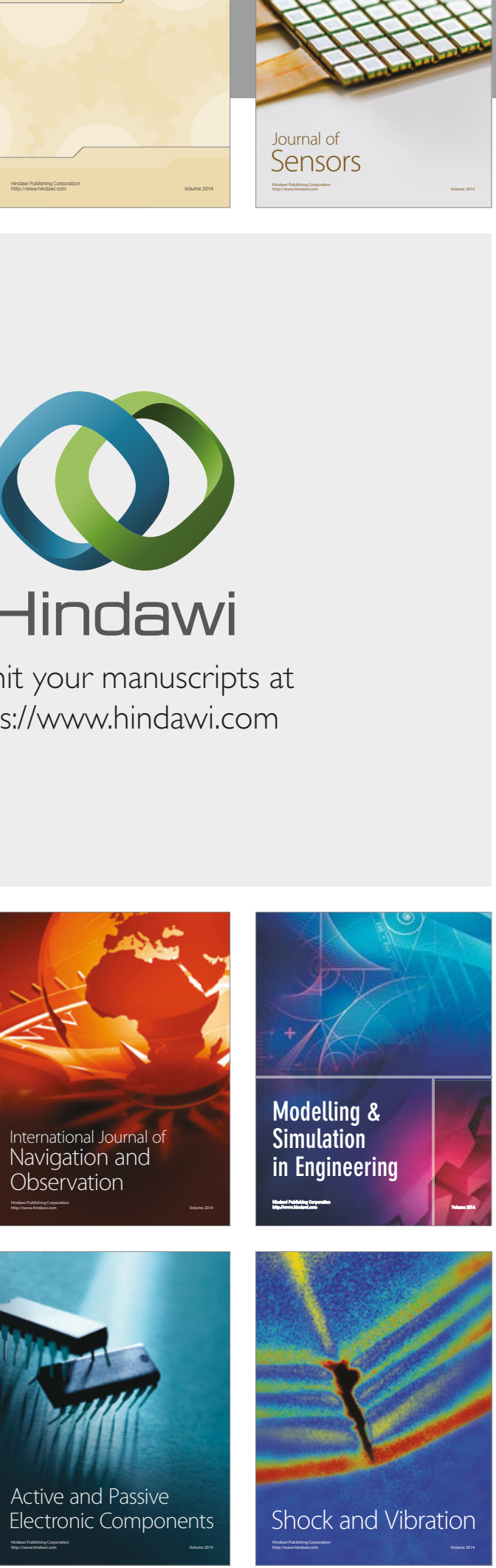
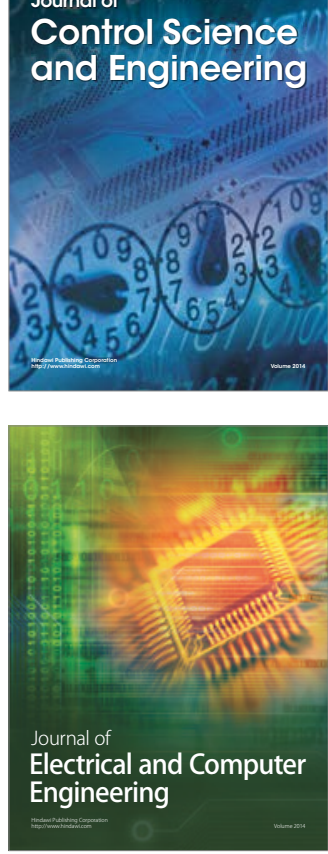

Distributed

Journal of

Control Science

and Engineering
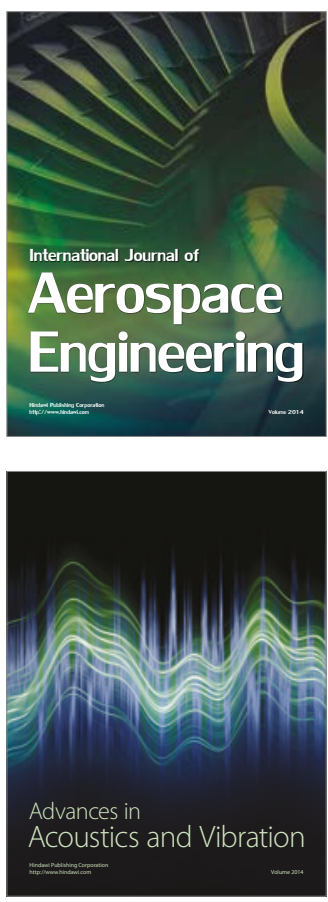

Sensor Networks 